\title{
Demonstration of amplitude-distortion correction by modal dispersal and phase conjugation
}

\author{
Yasuo Tomita, Kazuo Kyuma, ${ }^{*}$ Ram Yahalom, and Amnon Yariv \\ California Institute of Technology, Pasadena, California 91125
}

Received May 26, 1987; accepted September 23, 1987

\begin{abstract}
We demonstrate experimentally and explain theoretically polarization-preserving imaging through a lossy amplitude-distorting medium. This is accomplished by propagating the beam, before its arrival at the lossy distorting medium, through a (multi) mode- and polarization-scrambling fiber and reflecting the signal, after it has passed the lossy distorting medium, from a photorefractive phase-conjugate mirror.
\end{abstract}

Most of the distortion-correction schemes that are based on phase-conjugate optics involve phase-distorting media. ${ }^{1}$ This is because true phase conjugation does not obtain in cases involving inhomogeneous losses since part of the spatial information is lost and is not available for a reconstruction. In this Letter we describe a method of recovering an original image, including its polarization state, that has propagated through a lossy distorter by using modal dispersal in a multimode fiber and a photorefractive phase-conjugate mirror. ${ }^{2-5}$ In this method, before its incidence upon the lossy distorting medium the image-bearing beam is intentionally mode dispersed by propagating through a (multi) mode- and polarization-scrambling fiber so that the field exiting the fiber has the original pictorial and polarization information spread among a large number of modes; this robustness enables it, within certain limits, to reconstruct the original field including the polarization. Note that the image transmission through a multimode fiber (which acts as a modal phase distorter) by phase conjugation was originally suggested by Yariv ${ }^{6,7}$ and demonstrated by others. ${ }^{8-10}$ In the present study, however, we use the multimode fiber as a way to achieve mode and polarization scrambling of the input information. First we shall consider the method theoretically, followed by a description of the experimental demonstration.

The method is illustrated in Fig. 1. The spatialand polarization-information-bearing field $\mathbf{E}^{(1)}$ is launched into a mode-dispersing medium (e.g., a multimode fiber in our experiment), where the input field initially excites a portion of the total number of modes $2 N$ in the mode-dispersing medium. These are redistributed, because of a strong mode coupling, among the total $x$-polarized modes $N$ and the total $y$-polarized modes $N$ during propagation. Adopting the same mathematical notations introduced in Refs. 3 and 4, the field $\mathbf{E}^{(2)}$ exiting the mode-dispersing medium is expressed by

$$
\mathbf{E}^{(2)}=M \mathbf{E}^{(1)}
$$

and

$$
M=\left[\begin{array}{ll}
M_{x x} & M_{x y} \\
M_{y x} & M_{y y}
\end{array}\right],
$$

where $M$ is a scattering matrix of rank $2 N$ for traveling forward along the mode-dispersing medium. If a phase-conjugate mirror (PCM), as usual, reflects only one transverse component, say, the $x$-polarized component of the incident field, then only the $x$-polarized component $\mathbf{E}_{x}^{(3)}$ following the distorting medium is phase conjugated, so that $\mathbf{E}^{(4)}=\left[\mathbf{E}_{x}^{(3)}\right]^{*}$. The lefttraveling field $\mathbf{E}^{(5)}$ reentering the mode-dispersing medium from the right can consequently be written as

$$
\mathbf{E}^{(5)}=r C\left[\mathbf{E}^{(2)}\right]^{*},
$$

and

$$
C=\left[\begin{array}{cc}
C_{x x} & 0 \\
0 & 0
\end{array}\right]
$$

where $r$ is the phase-conjugate reflectivity of the PCM and $C$ accounts for the modal loss and mixing of the incoming field by the distorting medium. In the present analysis $C$ has the form of Eq. (4), where $C_{x x}$ is an $N \times N$ submatrix that accounts for the elimination of the $y$-polarized field and the modal loss and mixing of the $x$-polarized field in the distorting medium. The form of $C$ also implies that the loss does not scramble the polarizations. In particular, we have $\left(C_{x x}\right)_{i j}=\delta_{i j}$ when only a polarizer (oriented to the $x$ direction) acts as the distorting medium. ${ }^{3-5}$ The left-traveling field $\mathbf{E}^{(6)}$ exiting the input plane is given by

$$
\mathbf{E}^{(6)}=r M^{\prime} C M^{*}\left[\mathbf{E}^{(1)}\right]^{*},
$$

where $M^{\prime}$ denotes a scattering matrix for traveling backward along the mode-dispersing medium. From Eqs. (2) and (4) we obtain

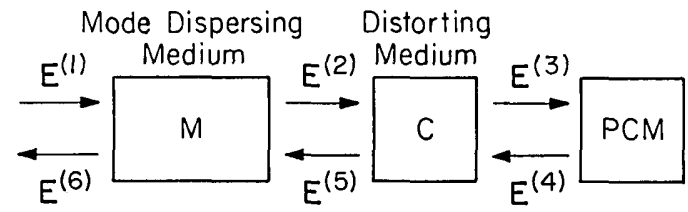

Fig. 1. A method to undo the lossy distortion effect by means of the tandem combination of the mode-dispersing medium (M) and the PCM. The lossy distortion due to the distorting medium (C) occurs between the M and the PCM. 


$$
M^{\prime} C M^{*}=\left[\begin{array}{ll}
M_{x x}^{\prime} C_{x x} M_{x x}^{*} & M_{x x}^{\prime} C_{x x} M_{x y}^{*} \\
M_{y x}^{\prime} C_{x x} M_{x x}^{*} & M_{y x}^{\prime} C_{x x} M_{x y}^{*}
\end{array}\right] .
$$

In the case of a large number of nonvanishing elements $\left(C_{x x}\right)_{i j}$, and because of the near equipartition of any modal energy and the modal averaging among the ensemble of modes through the strong mode coupling in the mode-dispersing medium, ${ }^{3-5}$ each element in Eq. (6) can be approximated by

$$
\left(M^{\prime} C M^{*}\right)_{i j} \rightarrow\left\{\begin{array}{cc}
\frac{1}{2 N} \sum_{k=1}^{N}\left(C_{x x}\right)_{k k} & \text { for } i=j . \\
O(1 / N) & \text { for } i \neq j
\end{array}\right.
$$

We therefore get

$$
\mathbf{E}^{(6)}=\frac{1}{2} r\left[\frac{1}{N} \sum_{k=1}^{N}\left(C_{x x}\right)_{k k}\right]\left[\mathbf{E}^{(1)}\right]^{*}+\mathbf{V},
$$

where $\mathbf{V}$ is a $2 N$ rank vector whose elements depend on the elements of $\mathbf{E}^{(1)}$ and $C_{x x}$ as well as $N$. We note that the first term on the right-hand side of Eq. (7) corresponds to the true phase-conjugate replica of the field $\mathbf{E}^{(1)}$ including polarization. The second term $\mathbf{V}$ corresponds to (depolarized) noise, and it can be shown both theoretically and experimentally that in the absence of the lossy distorter [i.e., $\left(C_{x x}\right)_{i j}=\delta_{i j}$ ] the total power of the noise is nearly the same as that of the true phase-conjugate beam and is distributed over all the fiber modes. However, this noise contribution can be negligible in the limit of small-numerical-aperture inputs. ${ }^{4,5}$ In the present case it is seen from Eq. (7) that the true phase conjugation is possible, within the above limits, even when a large number of modes are lost by the distorting medium as long as the near equipartition of modal energy in the mode-dispersing medium is satisfied so that the input information is distributed equally among all the modes.

In the experiment shown in Fig. 2, a knife edge was used as the simulated amplitude distorter (or medium) in order to eliminate some portions of the input information. In Fig. 2(a) a transparency $\mathrm{T}$ containing pictorial information was illuminated by the $x$-polarized beam from the multilongitudinal-mode argon-ion laser $(\lambda=514.5 \mathrm{~nm})$. The image-bearing beam $\mathbf{E}^{(1)}(5$ $\mathrm{mm}$ in diameter) was launched into a multimode (100$\mu \mathrm{m}$ core-diameter, 0.29 N.A., 5-m-long) graded-index fiber with about $0.036-N$.A. input by using an aperture AP and an $f=70 \mathrm{~mm}$ lens L1. With this small N.A. of the input compared with the fiber N.A., the noise contribution [corresponding to $\mathrm{V}$ in Eq. (7)] is negligible for the formation of the true phase conjugation. The knife edge $\mathrm{F}$ was placed in the image plane of the fiber end and eliminated some portions of the outcoupled beam $\mathbf{E}^{(2)}$. In this case the microscope objective L2 of 0.40 N.A. covering 0.29 N.A. of the fiber was used to image the fiber end through a polarizer $\mathrm{P}$ oriented to the $x$ direction. A poled $\mathrm{BaTiO}_{3}$ crystal was used as a self-pumped PCM, and its phase-conjugate reflectivity was $35 \%$ for the incident power of 3 $\mathrm{mW}$ on the crystal. The reflected beam $\mathbf{E}^{(4)}$ from the input end of the fiber is picked off by a nonpolarizing beam splitter BS and forms the reconstructed image on the screen S. The analyzer A was used to partly eliminate the noise contribution.

In Fig. 2(b) F was placed in the far-field plane of the fiber end for a comparison of the results with those of the former configuration. In this case the out-coupled beam $\mathbf{E}^{(2)}$ was quasi-collimated (about $3 \mathrm{~mm}$ in diameter) by L2, and some portions of the quasi-collimated beam were eliminated by $\mathrm{F}$.

First we measured the Stokes parameters $\left(s_{0}, s_{1}, s_{2}\right.$, $s_{3}$ ) of the fields $\mathbf{E}^{(1)}, \mathbf{E}^{(2)}$, and $\mathbf{E}^{(4)}$ so that the degree of polarization ${ }^{11} P=\left(s_{1}{ }^{2}+s_{2}{ }^{2}+s_{3}^{2}\right)^{1 / 2} s_{0}$ and the degree of polarization recovery $p=s_{1} / s_{0}$ could be calculated. ${ }^{4}$ The $x$-polarized $(P, p>0.99)$ uniform beam was used as the input field $\mathbf{E}^{(1)}$ together with the configuration shown in Fig. 2(a). Because of the strong mode coupling in the fiber the field $\mathbf{E}^{(2)}$ exhibited speckled spatial structures and nearly complete depolarization $(P, p<0.09)$. Figure 3 shows $P$ and $p$ of the field $\mathbf{E}^{(4)}$ at $\mathrm{S}$ in the absence of $\mathrm{A}$ as a function of various transmission losses due to $\mathrm{F}$. It is seen that $P$ and $p$ are almost equal for all losses and both decrease as the loss increases, which shows that the original polarization recovery deteriorated gradually because of the relative increase of the depolarized noise as the loss increases. This may indicate that the equipartition of the input

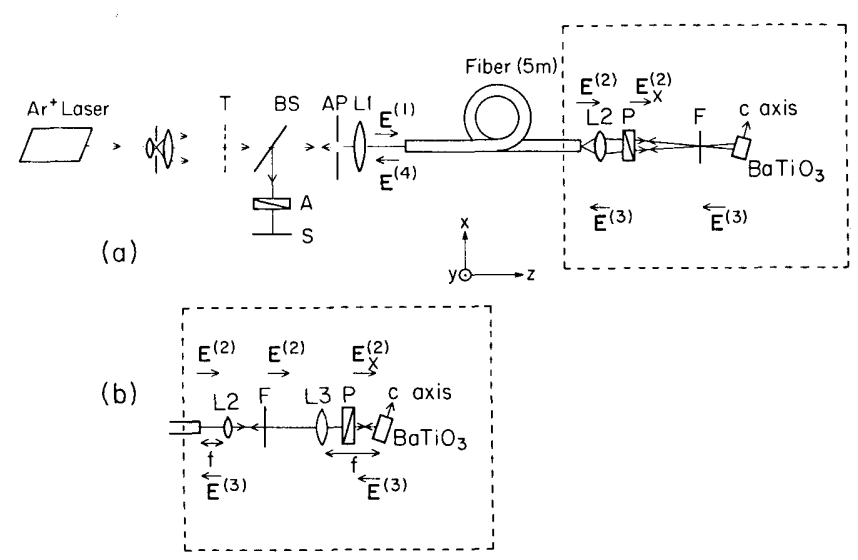

Fig. 2. The experimental arrangement. In (a) the outcoupled beam $\mathbf{E}^{(2)}$ is imaged onto F. In (b) $\mathbf{E}^{(2)}$ is quasicollimated onto $\mathrm{F}$.

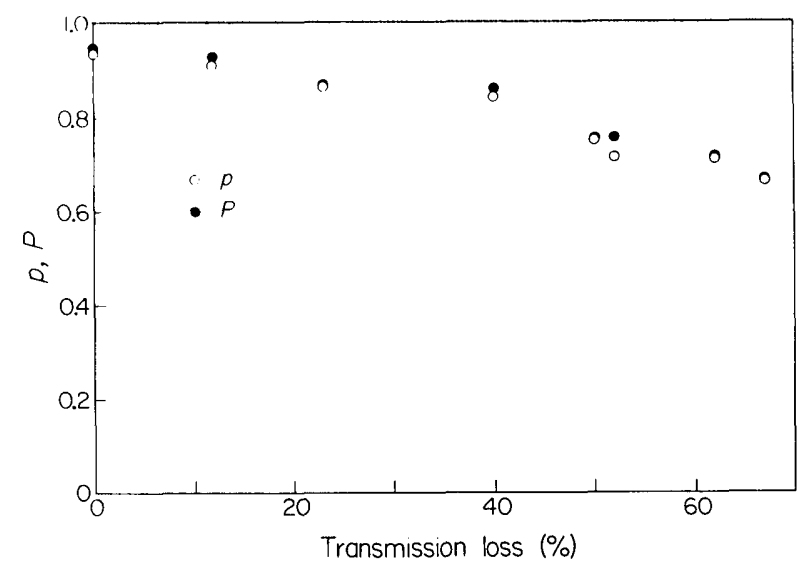

Fig. 3. The degree of polarization $P$ and the degree of polarization recovery $p$ of the field $\mathbf{E}^{(4)}$ at $S$ as a function of the transmission loss due to $\mathrm{F}$ for the linearly $x$-polarized input beam. 

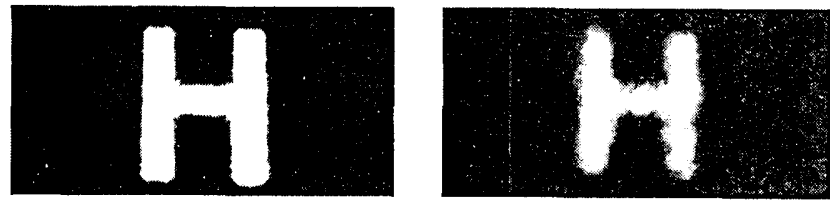

(a)

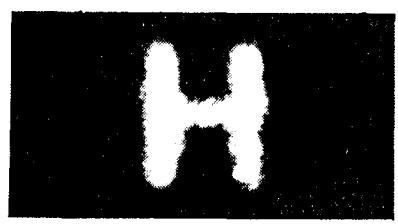

(b)

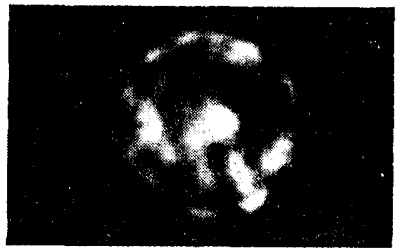

(c)

(d)

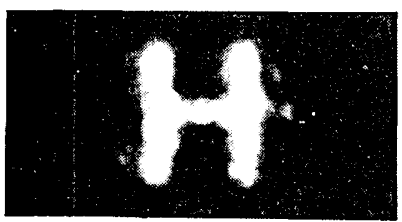

(e)

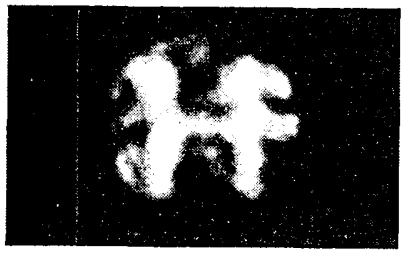

(f)

Fig.4. (a) Input image. (b) Phase-conjugate image of the $x$ polarization without $\mathrm{F}$ (12 times the intensity-attenuated image). (c) Phase-conjugate image of the $y$ polarization without $\mathrm{F}$. (d)-(f) Phase-conjugate images of the $x$ polarization with various transmission losses due to F: (d) loss $23 \%$ (12 times the intensity-attenuated image), (e) loss $53 \%$ ( 2 times the intensity-attenuated image), (f) loss $68.5 \%$.

information among all the modes in the fiber used is incomplete. Nonetheless, we see that the polarization recovery is still about 0.66 , even in the case of the largest loss of $67 \%$, i.e., $66 \%$ of the reflected beam power is in the true phase-conjugate beam and the remainder is in the depolarized noise. Figure 4(a) is the $x$-polarized input when the transparency of a picture of the letter $\mathrm{H}$ is used. Figures 4(b)-4(f) show the phase-conjugate images at $\mathrm{S}$ when the configuration shown in Fig. 2(a) is used. Figures 4(b) and 4(c) show the $x$-polarized component and the $y$-polarized component of the phase-conjugate image at $\mathrm{S}$ without $\mathrm{F}$, respectively. It is seen that the phase-conjugate image preserves its original polarization and spatial structure. Figures 4(d)-4(f) show the phase-conjugate images at $\mathrm{S}$ with various transmission losses due to F: Fig. 4(d) 23\%, Fig. 4(e) 53\%, and Fig. 4(f) $68.5 \%$. It is clear from these figures that although the intensities of the phase-conjugate images decrease as the transmission loss increases, the phase-conjugate replica of the input image can be reconstructed. However, we see the apparent degradation of the spatial structure of the phase-conjugate image shown in Fig. 4(f). This may also be attributed, as mentioned above, to the incomplete equipartition of the input information among all the modes in the fiber. Therefore above a certain limit of the loss by F [the case of Fig. 4(f)] the reconstructed image bears less information than the original image, resulting in the apparent degradation. This effect may be analogous to the case of image reconstruction in holography with a diffused signal, ${ }^{11}$ when the resolution in the reconstructed image decreases as the fragment of hologram becomes smaller. In our case the degradation depends strongly on the modal-scrambling nature of the fiber. We could also obtain the same results with the configuration shown in Fig. 2(b). This fact indicates that, although the mode scrambling of the input information in the fiber used is not complete, the input information is redistributed among a sufficiently large number of modes (viz., spatial frequencies) of the out-coupled field from the fiber, and therefore the present scheme is almost insensitive to the position of the distorter between the fiber and the PCM. ${ }^{12}$

In conclusion, we have demonstrated polarizationpreserving imaging through a lossy amplitude distortion using modal dispersal and phase conjugation. Our experiments have confirmed that the polarization and spatial information of the input is distributed among a large number of fiber modes through the strong mode coupling in a fiber and can be reconstructed following phase conjugation in spite of spatially lossy distortions as well as the elimination of one polarization component. It has also been found that the present scheme is insensitive to the position of the distorter.

This research was supported by the U.S. Air Force Office of Scientific Research and by the U.S. Army Research Office, Durham, North Carolina.

* Present address, Central Research Laboratory, Mistubishi Electric Corporation, 8-1-1 TsukaguchiHonmachi, Amagasaki, Hyogo, 661 Japan.

\section{References}

1. R. A. Fisher, ed., Optical Phase Conjugation (Academic, New York, 1983), and references therein.

2. K. Kyuma, A. Yariv, and S.-K. Kwong, Appl. Phys. Lett. 49, 618 (1986).

3. A. Yariv, Y. Tomita, and K. Kyuma, Opt. Lett. 11, 809 (1986).

4. Y. Tomita, R. Yahalom, and A. Yariv, Opt. Lett. 12, 1017 (1987).

5. Y. Tomita, R. Yahalom, and A. Yariv, "Theory of polarization and spatial information recovery by modal dispersion," J. Opt. Soc. Am. B (to be published).

6. A. Yariv, J. Opt. Soc. Am. 66, 301 (1976).

7. A. Yariv, Appl. Phys. Lett. 28, 88 (1976).

8. G. J. Dunning and R. C. Lind, Opt. Lett. 7, 558 (1982).

9. B. Fisher and S. Sternklar, Appl. Phys. Lett. 46, 113 (1985).

10. P. H. Beckwith, I. McMichael, and P. Yeh, Opt. Lett. 12, 510 (1987).

11. R. J. Collier, C. B. Burckhardt, and L. H. Lin, Optical Holography (Academic, New York, 1971), p. 197.

12. It should be noted that since the experiment is made for the distorter placed on both the image plane and the farfield plane of the fiber end, the phase-conjugate images obtained here are not a consequence of simple Fourier filtering of the input image. 